Originalien

Nervenarzt 2020 $91: 337-342$ https://doi.org/10.1007/s00115-020-00885-1 Online publiziert: 6. März 2020

(c) Der/die Autor(en) 2020

L. Klingelhoefer' $\cdot$ W. Jost ${ }^{2} \cdot$ P. Odin ${ }^{3} \cdot$ A. Storch ${ }^{4,5} \cdot$ K. Ray Chaudhuri ${ }^{6}$.

H. Reichmann ${ }^{1}$

${ }^{1}$ Klinik und Poliklinik für Neurologie, Technische Universität Dresden, Dresden, Deutschland

${ }^{2}$ Parkinson-Klinik Ortenau, Wolfach, Deutschland

${ }^{3}$ Division of Neurology, Skåne University Hospital, Lund University, Lund, Schweden

${ }^{4}$ Klinik und Poliklinik für Neurologie, Universität Rostock, Rostock, Deutschland

${ }^{5}$ Deutsches Zentrum für Neurodegenerative Erkrankungen (DZNE) Rostock/Greifswald, Rostock, Deutschland

${ }^{6}$ National Parkinson Foundation International Centre of Excellence, Department of Neurology, King's

College Hospital, Denmark Hill, London, Großbritannien

\title{
Dystonia Non-Motor Symptoms Questionnaire (DNMSQuest) zur Erhebung nichtmotorischer Symptome bei Dystonie
}

\section{Interkulturelle Adaptation in deutscher Sprache}

\begin{abstract}
Die Kenntnis nichtmotorischer Symptome bei Patienten mit Dystonie, deren Erhebung und Behandlung im klinischen Alltag sind wichtig, da diese neben den motorischen Beschwerden einen relevanten Einfluss auf die Lebensqualität haben. Der Dystonia Non-Motor Symptoms Questionnaire als einfacher und schnell durch die Patienten selbst auszufüllender Fragebogen erfasst 7 verschiedene Domänen (Schlaf, autonome Symptome, Fatigue, emotionales Wohlbefinden, Stigma, Aktivitäten des täglichen Lebens, sensorische Symptome) und kann zum Erreichen eines ganzheitlichen und erfolgreichen Behandlungskonzeptes bei Patienten mit Dystonie beitragen.
\end{abstract}

\section{Hintergrund und Fragestellung}

Dystonien gehören zu den häufigsten Bewegungsstörungen. Hierbei stellt die zervikale Dystonie zum einen die häufigste Form der fokalen Dystonien dar und ist mit einer Manifestation im höheren Erwachsenenalter um die 5. Lebensdekade insbesondere bei weiblichen Patienten die am häufigsten zu behandelnde Dystonie der Erwachsenenneurologie [1-3]. Neben den offensichtlichen motorischen Beschwerden bei Patienten mit Dystonien sind in den letzten Jahren vermehrt die nichtmotorischen Symptome (NMS) in den Fokus der Wissenschaft gerückt. Mit der zervikalen Dystonie assoziierte Schmerzen, psychopathologische Begleitsymptome, Schlafstörungen sowie Alltagseinschränkungen wurden in diesem Kontext beschrieben [4-8]. Bisherige Untersuchungen unterscheiden sich deutlich in der Häufigkeit der verschiedenen NMS. Auch ist ein ganzheitliches Bild der NMS bei Patienten mit zervikaler Dystonie nicht bekannt. Ein relevanter Grund hierfür ist, dass es keinen Fragebogen zur Erfassung von NMS bei Patienten mit Dystonie bzw. bestimmten Dystonieformen gibt. Nichtmotorische Symptome werden teilweise im Rahmen validierter motorischer Beurteilungsskalen wie z.B. Schmerz im TWSTRS Teil 3 mit erfasst [9]. Der Kraniozervikale Dystonie Fragebogen (CDQ-24; [10]) erfasst krankheitsspezifisch für Patienten mit kraniozervikaler
Dystonie ausgewählte NMS wie Stigma, emotionales Wohlbefinden und Schmerz zur Abschätzung der Lebensqualität, aber ein ganzheitliches NMSBild entsteht nicht. Auch ist die notwendige Zeitdauer zum Ausfüllen des CDQ-24 in Bezug auf die Möglichkeiten im klinischen Alltag relativ lang. Einen Screeningfragebogen zur krankheitssowie symptomspezifischen Erfassung nichtmotorischer Beschwerden bei $\mathrm{Pa}$ tienten mit kraniozervikaler Dystonie, welcher in den klinischen Alltag effizient und mit Erhalt valider Ergebnisse integriert werden kann, gibt es bisher nicht. Diese Lücke soll durch den Dystonia Non-Motor Symptoms Questionnaire (DNMSQuest) geschlossen werden.

Von anderen neurologischen Bewegungsstörungen, wie dem idiopathischen Parkinson-Syndrom, ist die Wichtigkeit von NMS, insbesondere auch im Hinblick auf den relevanten Einfluss auf die gesundheitsbezogene Lebensqualität gut bekannt $[11,12]$. Somit ist ein Fragebogen wie der DNMSQuest zur Erhebung von NMS bei Patienten mit Dystonie sowohl für die alltägliche Betreuung dieser Patienten zum Erkennen und ent- 
sprechenden individuellen Behandeln der krankheitsspezifischen Symptome als auch aufgrund der Einflüsse dieser krankheitsspezifischen Symptome auf die gesundheitsbezogene Lebensqualität sowie für eine Verbesserung des wissenschaftlichen Verständnisses dieser Erkrankung von Bedeutung. Der DNMSQuest kann dazu beitragen, die Häufigkeit von NMS sowie deren Auswirkungen auf die gesundheitsbezogene Lebensqualität bei Patienten mit kraniozervikaler Dystonie zu untersuchen, um diese im klinischen Alltag effizienter diagnostizieren und behandeln $\mathrm{zu}$ können.

\section{Methoden}

Die interkulturell adaptierte Version des DNMSQuest in deutscher Sprache basiert auf den etablierten und anerkannten internationalen Standards zum Vorgehen der interkulturellen Adaptation selbst auszufüllender Patientenfragebögen [13, 14]. Zunächst erfolgte die Übersetzung der englischen Originalfassung durch zwei bilinguale Neurologen mit Schwerpunkt im Bereich der Bewegungsstörungen (HR, AS) getrennt und unabhängig voneinander ins Deutsche. Durch einen weiteren Neurologen (LK) wurden die beiden Fassungen abgeglichen und eine von beiden Übersetzern akzeptierte Konsensusfassung erstellt. Diese vorläufige Fassung der deutschen Übersetzung wurde bei 10 Patienten mit zervikaler Dystonie im Rahmen der Dystonie-Sprechstunde in der Klinik und Poliklinik für Neurologie des Universitätsklinikums Dresden eingesetzt und mit den entsprechenden Patienten über die Verständlichkeit des Erklärungstextes sowie der Fragen und mögliche Missverständnisse beraten (LK). Rückmeldungen von Patienten wurden in der dann ersten deutschen Fassung berücksichtigt. Diese Konsensusfassung wurde anschließend von zwei anderen bilingualen Neurologen mit Schwerpunkt im Bereich der Bewegungsstörungen (PO, WJ) getrennt und unabhängig voneinander ins Englische als Originalsprache zurückübersetzt und hieraus wurde wiederum eine englische Konsensusfassung erstellt (LK). Diese englische Konsen- susfassung wurde von einem weiteren Neurologen mit Schwerpunkt im Bereich der Bewegungsstörungen (KRC), welcher maßgebend an der Entwicklung der englischen Originalfassung des DNMSQuest beteiligt war [15-17], mit der englischen Originalfassung abgeglichen. Hier erfolgte insbesondere die Prüfung auf relevante sprachliche und inhaltliche Abweichungen der Fragebögen voneinander. Anschließend erfolgte eine entsprechende finale Anpassung der deutschen Version, sodass sie im Konsens aller Übersetzer sprachlich akzeptabel und in der Rückübersetzung ins Englische eindeutig war. Es resultierte der endgültige adaptierte DNMSQuest in deutscher Sprache (๑ Abb. 1).

Diese finale deutsche Version des DNMSQuest wurde im Rahmen der international durchgeführten multizentrischen Validierungsstudie des DNMSQuest in englischer sowie deutscher Sprache [18] in den deutschen Studienzentren (Klinik und Poliklinik für Neurologie der Technischen Universität Dresden, Klinik und Poliklinik für Neurologie der Universität Rostock, Klinik für Neurologie des Klinikums Chemnitz) genutzt. Das entsprechende Studienprotokoll wurde durch die zuständigen Ethikkommissionen geprüft und genehmigt (Dresden and Chemnitz: EK60022015, Rostock: A2016-0159). Hier wurden 130 Patienten mit zervikaler Dystonie sowie 48 gesunde Kontrollpersonen mit der deutschen Version des DNMSQuest untersucht; entsprechende Einverständniserklärungen der Studienteilnehmer lagen vor.

\section{Ergebnisse}

Die Testung des DNMSQuest an Patienten mit zervikaler Dystonie im Rahmen der interkulturellen Adaptation sowie die entsprechenden Übersetzungen (Konsensusbildung einer ersten deutschen Version und deren Rückübersetzung ins Englische) zeigten keine relevanten Verständnisschwierigkeiten bzw. sprachliche Unterschiede im Erklärungstext des Fragebogens.

Bei 7 der 14 Fragen ergaben sich Abweichungen im Vergleich zwischen den beiden deutschen Übersetzungen der englischen Originalversion des DNMSQuest. Hierbei handelte es sich vor allem um sprachliche Formulierungsunterschiede wie z.B. Frage 2 „Probleme ein- oder durchzuschlafen“ bzw. „Einoder Durchschlafprobleme“, Frage 3 „Benommenheit" bzw. „Kopfleere“, Frage 4 „Energiemangel“ bzw. „Energielosigkeit", Frage 5 „,ohne erkennbaren Grund“ bzw. „unbegründet" sowie „ängstlich“ bzw. „verängstigt“, Frage 6 „deprimiert“ bzw. „depressiv“, Frage 14 „Gleichgewichtsprobleme“ bzw. „Gangunsicherheit“. Auch bei der Rückübersetzung der ersten deutschen Konsensusfassung ins Englische stellten sich bei 5 der 14 Fragen sprachliche Formulierungsunterschiede dar, wie z. B. Frage 1 „not recovered“ bzw. „unrested“, Frage 2 „staying asleep“ bzw. „sleeping though till morning", Frage 3 „vertigo“ bzw. „dizziness“, Frage 7 „suffer from a loss of self-confidence" bzw. „lack self-confidence“. Bei Abgleich der englischen Konsensusfassung mit der englischen Originalfassung durch KRC, der maßgeblich an der Entwicklung der englischen Originalversion des DNMSQuest beteiligt war, ergaben sich keine inhaltlichen Abweichungen.

Die so entwickelte finale Fassung des interkulturell adaptierten DNMSQuest in deutscher Sprache ist in - Abb. 1 dargestellt. Weder bei der ersten Anwendung dieser deutschen Konsensusvariante bei einer kleinen Gruppe von Patienten mit zervikaler Dystonie noch im Rahmen der anschließend erfolgten breiten Anwendung zur Validierung des DNMSQuest und auch im Rahmen der bereits weitergeführten Anwendung in der klinischen Routine der DystonieSprechstunden ergaben sich Hinweise für systematische Verständnisschwierigkeiten oder Missverständnisse von Seiten der Patienten und der gesunden Kontrollpersonen. Weiterhin zeigte sich eine hohe Akzeptanz und Bereitschaft, den Fragebogen bereits im Rahmen der Wartezeit auf den Sprechstundentermin auszufüllen.

\section{Diskussion}

Die Erstellung der deutschen Fassung des DNMSQuest erfolgte entsprechend den internationalen Vorgaben zur interkul- 
Nervenarzt 2020 -91:337-342 https://doi.org/10.1007/s00115-020-00885-1

(c) Der/die Autor(en) 2020

L. Klingelhoefer · W. Jost · P. Odin · A. Storch · K. Ray Chaudhuri · H. Reichmann

\section{Dystonia Non-Motor Symptoms Questionnaire (DNMSQuest) zur Erhebung nichtmotorischer Symptome bei Dystonie. Interkulturelle Adaptation in deutscher Sprache}

\section{Zusammenfassung}

Hintergrund. Nichtmotorische Symptome

(NMS) bei Patienten mit Dystonie haben einen

relevanten Einfluss auf die Lebensqualität.

Ein rein auf NMS bei Dystonie bezogenes

Messinstrument existiert nicht.

Ziel. Die validierte deutsche Version des

Dystonia Non-Motor Symptoms Questionnaire

(DNMSQuest) zur Erhebung von NMS bei

kraniozervikaler Dystonie wird hier vorgestellt.

Methoden. Der DNMSQuest in deut-

scher Sprache wurde nach international

anerkannten Standards zum Vorgehen

der interkulturellen Adaptation selbst

auszufüllender Patientenfragebögen erstellt.

So erfolgte die Übersetzung des englischen

Originalfragebogens ins Deutsche sowie die

Rückübersetzung unabhängig voneinander durch insgesamt vier bilinguale Neurologen

mit Schwerpunkt Bewegungsstörungen.

Von einem weiteren Neurologen wurde

jeweils eine von den Übersetzern akzeptierte Konsensusfassung erstellt. Der englische Originalfragebogen wurde mit der rückübersetzten englischen Version von einem Neurologen mit maßgeblicher Beteiligung an der Entwicklung des Originalfragebogens auf sprachliche und inhaltliche Abweichungen kontrolliert. Die finale deutsche Version wurde bei 130 Patienten mit zervikaler Dystonie sowie 48 gesunden Kontrollen bei der internationalen, multizentrischen Validierungsstudie angewendet.
Ergebnisse. Es entstand eine interkulturell adaptierte, in deutscher und englischer Sprache validierte Fassung des DNMSQuest. Diskussion. Der DNMSQuest kann die aktuell bestehende Lücke eines validierten, krankheitsspezifischen, durch die Patienten selbst auszufüllenden, kurzen, umfassenden Fragebogen zur Erhebung von NMS bei zervikaler Dystonie schließen. Er kann einfach in den klinischen Alltag integriert werden und empfiehlt sich für die klinische Praxis sowie zur Anwendung in klinischen Studien.

Schlüsselwörter

Dystonia Non-Motor Symptoms Questionnaire · DNMSQuest · Dystonie · Nichtmotorische Symptome · Fragebogen

\section{Dystonia Non-Motor Symptoms Questionnaire (DNMSQuest) for assessment of non-motor symptoms in dystonia. Intercultural adaptation in the German language}

\section{Abstract}

Background. Non-motor symptoms (NMS) in patients with dystonia have a relevant impact on health-related quality of life; however, a comprehensive easy to use NMS assessment tool for clinical bedside use is currently not available.

Objective. The validated German version of the dystonia non-motor symptoms questionnaire (DNMSQuest) for assessing NMS in craniocervical dystonia is presented. Methods. The DNMSQuest in the German language was developed based on internationally recognized standards for intercultural adaptation of self-completed patient questionnaires. Translation of the original English questionnaire into the German language as well as back translation to English was carried out independently by four bilingual specialists in neurological movement disorders. In each case a consensus version accepted by each translator was created by another neurologist. The back translated English version was compared with the original English questionnaire for relevant linguistic and content discrepancies by a neurologist who was significantly involved in the development of the original questionnaire. The final German version was used in 130 patients with cervical dystonia and 48 healthy controls in an international, multicenter validation study.

Results. An interculturally adapted validated version of the DNMSQuest in the German and English languages was developed for rapid bedside assessment and evaluation of NMS in cervical dystonia.

Conclusion. The DNMSQuest successfully bridges the current gap of a validated disease-specific, patient self-administered, short, comprehensive questionnaire for NMS assessment in routine clinical practice in craniocervical dystonia. It is envisaged that this tool will be useful for the clinical practice and trials.

Keywords

Dystonia Non-Motor Symptoms Questionnaire · DNMSQuest · Dystonia - Non-motor symptoms · Questionnaire turell adaptierten Übersetzung patientenbezogener Fragebögen [13, 14]. Diese deutsche Version wurde im Rahmen der international erfolgten multizentrischen Validierungsstudie des DNMSQuest in den deutschen Studienzentren genutzt [18]. Die entsprechenden klinimetrischen Eigenschaften des DNMSQuest sind in der Validierungsstudie dargestellt [18]. Hier konnte gezeigt werden, dass der DNMSQuest ein valides, robustes und gut reproduzierbares Instrument mit angemessenen klinimetrischen Werten zur Erfassung von NMS bei Patienten mit zervikaler Dystonie ist.

Der DNMSQuest ist ein vom Patienten selbst auszufüllender Fragebogen (Dauer ca. $5 \mathrm{~min}$ ), der in 14 binären „Ja/Nein“-Fragen nichtmotorische Symptome, die innerhalb der letzten vier Wochen aufgetreten sind, erfragt. Die 14 Fragen können auf 7 Domänen auf- geteilt werden: Schlaf (2 Fragen, Frage 1 und 2), autonome Symptome (1 Frage, Frage 3), Fatigue (1 Frage, Frage 4), emotionales Wohlbefinden (3 Fragen, Frage 5, 6, 8), Stigma (1 Frage, Frage 7), Aktivitäten des täglichen Lebens (4 Fragen, Frage 9, 11, 12, 14), sensorische Symptome (2 Fragen, Frage 10, 13). Die Gesamtpunkzahl (minimal 0 Punkte bis maximal 14 Punkte) ergibt sich durch Summieren der „Ja“-Antworten, wobei 
Fragebogen zu nicht motorischen Symptomen bei Dystonien: „Dystonia Non-Motor Symptoms Questionnaire (DNMSQuest)“"

Eine Reihe von Problemen ist unten angeführt. Bitte kreuzen Sie das Feld ${ }_{\Perp} \mathrm{Ja}^{\text {“ }}$ an, wenn Sie das beschriebene Symptom während des letzten Monats erlebt haben. Wenn bei Ihnen das jeweilige Problem im Laufe des letzten Monats nicht aufgetreten ist, kreuzen Sie bitte das Feld "Nein" an. Sie sollten auch dann "Nein" antworten, wenn Sie die Symptome in der Vergangenheit, aber nicht während des letzten Monats hatten.

\begin{tabular}{|c|c|c|}
\hline \multirow{2}{*}{ 1. Fühlen Sie sich NICHT erholt, obwohl Sie nachts schliefen? } & $\mathrm{Ja}$ & Nein \\
\hline & & \\
\hline 2. Haben Sie Probleme ein- oder durchzuschlafen? & & \\
\hline 3. Leiden Sie unter Benommenheit oder Schwindel? & & \\
\hline 4. Schränken Müdigkeit oder Energiemangel Ihre Tagesaktivitäten ein? & & \\
\hline 5. Fühlen Sie sich ohne erkennbaren Grund nervös, besorgt oder ängstlich? & & \\
\hline 6. Fühlen Sie sich traurig oder deprimiert? & & \\
\hline $\begin{array}{l}\text { 7. Leiden Sie unter einem Verlust an Selbstvertrauen aufgrund des Stigma durch die } \\
\text { erkennbare (Hals)Dystonie? }\end{array}$ & & \\
\hline $\begin{array}{l}\text { 8. Sind Sie nicht mehr schwingungsfähig, das heißt ohne die normalen Höhen und } \\
\text { Tiefen der Stimmungslage? }\end{array}$ & & \\
\hline 9. Haben Sie Probleme beim Essen, zum Bespiel beim Kauen oder Schlucken? & & \\
\hline $\begin{array}{l}\text { 10. Haben Sie in oder in der Nähe der Körperregion, in der sich Ihre Dystonie zeigt, } \\
\text { unangenehme Empfindungen wie Taubheit, Kribbeln oder Stechen? }\end{array}$ & & \\
\hline 11. Haben Sie Probleme beim Sprechen? & & \\
\hline $\begin{array}{l}\text { 12. Beeinflusst Ihre Dystonie Ihr Sehvermögen, beispielweise wenn sich ihr Kopf zur } \\
\text { Seite dreht? }\end{array}$ & & \\
\hline $\begin{array}{l}\text { 13. Leiden Sie unter Schmerzen (schmerzhafter Verspannung) im Bereich Ihrer } \\
\text { Dystonie oder im Umfeld dieser Körperregion (ohne andere Gründe in dieser } \\
\text { Körperregion für solche Schmerzen)? }\end{array}$ & & \\
\hline $\begin{array}{l}\text { 14. Leiden Sie unter irgendwelchen Schwierigkeiten beim Gehen oder Gleichgewichts- } \\
\text { problemen? }\end{array}$ & & \\
\hline
\end{tabular}

Abb. $1 \triangleleft$ Der Dystonia Non-Motor Symptoms Questionnaire (DNMSQuest) in deutscher Sprache zur Erhebung nichtmotorischer Symptome bei Patienten mit kraniozervikaler Dystonie eine höhere Gesamtpunktzahl dementsprechend eine größere Anzahl an NMS bedeutet. Ein Nachweis von 5 NMS wurde als relevante Belastung eingeordnet [18].

Da es sich beim DNMSQuest um einen Screeningfragebogen mit rein qualitativen Fragen zu einzelnen NMS zur schnellen Erfassung im klinischen Alltag handelt, ist keine Abschätzung über die Häufigkeit des Auftretens und die jeweilige Schwere der NMS möglich. Die hohe Validität des DNMSQuest, die gute Akzeptanz bei Patienten mit zervikaler Dystonie sowie der geringe Zeitaufwand zum Ausfüllen des DNMSQuest durch die Patienten sowie auch für die Auswertung durch den Arzt ermöglichen jedoch eine sinnvolle Anwendung zum Screening auf die meisten NMS in der klinischen Praxis. Eine nähere Einordnung und die Einschätzung der Therapiebedürftigkeit der NMS sollten anschließend fokussiert und individuell im Arztgespräch erfolgen. Neben der klinisch praktischen Anwendung besteht mit dem DNMSQuest nun erstmalig auch ein Fragebogen, welcher zwar nur quantitativ, aber ganzheitlich wichtige NMS bei Patienten mit kraniozervikaler Dystonie erfasst und hiermit auch zur Anwendung für klinische Studien geeignet ist. Aktuell verfügbare Dystoniebeurteilungsskalen fokussieren sich vorwiegend auf die motorische Symptomschwere der verschiedenen anatomisch betroffenen Körperregionen sowie entsprechende Provokationsfaktoren und die Dauer der Dystonie (TWSTRS, Unified Dystonia Rating Scale) [9, 19]. Einzelne NMS wie z. B. Schmerz werden sehr detailliert abgefragt (TWSTRS Teil III; [9]) oder auch der Effekt auf die Alltagsaktivitäten [9] oder die gesundheitsbezogene Lebensqualität [10]. Ein umfassendes, in den klinischen Alltag einfach integrierbares, rein auf nichtmotorische Symptome bei Dystonie bezogenes Messinstrument gibt es allerdings nicht [20]. Der DNMSQuest kann somit diese aktuell bestehende Lücke eines validierten, krankheitsspezifischen, durch die $\mathrm{Pa}$ tienten selbst auszufüllenden, kurzen, umfassenden und in den klinischen Alltag einfach $\mathrm{zu}$ integrierenden Fragebogen zur Erhebung nichtmotorischer Symptome bei Dystonie schließen. Für die klinische Praxis ist dies insbesondere relevant, da die Bewertung nichtmotorischer wie auch motorischer Symptome 
individuell bei jedem Dystoniepatienten zum Erreichen eines ganzheitlichen und erfolgreichen Therapiekonzeptes empfohlen wird [21]. Weiterhin konnte gezeigt werden, dass die zervikale Dystonie einen vergleichbaren negativen Effekt auf die gesundheitsbezogene Lebensqualität wie andere chronische neurologische Erkrankungen, z. B. die Multiple Sklerose, die Parkinson-Erkrankung oder der Schlaganfall, hat $[22,23]$. NMS spielen hier eine entscheidende Rolle [22, 24, 25]. Bei der Parkinson-Erkrankung hat sich bereits gezeigt, dass die Erfassung von NMS neben den obligaten motorischen Symptomen $\mathrm{zu}$ Vorteilen für die Patienten führt. Zum einen durch das Schaffen von Bewusstsein und Wissen über diesen Teil der Erkrankung sowohl bei den Patienten als auch bei den behandelnden Ärzten, zum anderen durch eine spezifische Behandlung bzw. Verlaufskontrolle der individuellen Beschwerden mit positiven Effekten auf die gesundheitsbezogene Lebensqualität $[12,26]$.

Ein validierter Fragebogen in deutscher Sprache zur schnellen und einfachen Erfassung und Bewertung von NMS bei Patienten mit kraniozervikaler Dystonie liegt nun mit dem DNMSQuest vor. Natürlich wird der DNMSQuest als rein quantitativer Fragebogen nicht allen Ansprüchen bezogen auf die Komplexität von NMS bei Dystonie gerecht. Er stellt aber einen entscheidenden Baustein dar, um über mehr Bewusstsein und Wissen sowohl klinisch als auch wissenschaftlich ein besseres Verständnis mit positiven Effekten in der Versorgung von Patienten mit zervikaler Dystonie zu erreichen.

\section{Fazit für die Praxis}

\section{- Der Dystonia Non-Motor Symp- toms Questionnaire (DNMSQuest) ist der erste spezifische, umfassende Fragebogen zur Erhebung nichtmo- torischer Symptome bei Patienten mit kraniozervikaler Dystonie. \\ - Er kann durch die Patienten selbst- ständig innerhalb von ca. 5 min im Rahmen der Wartezeit auf den Sprechstundentermin ausgefüllt werden.}

- Die 14 binären „Ja/Nein“-Fragen geben dem Arzt Auskunft über 7 verschiedene nichtmotorische Symptomdomänen (Schlaf, autonome Symptome, Fatigue, emotionales Wohlbefinden, Stigma, Aktivitäten des täglichen Lebens/ Alltagskompetenz, sensorische Symptome).

- Der DNMSQuest ist valide, robust sowie einfach in den klinischen Alltag zu integrieren.

- Der DNMSQuest empfiehlt sich für die klinische Praxis sowie zur Anwendung in klinischen Studien. - Die sprachlich validierte deutsche Fassung wird in dieser Arbeit vorgestellt.

\section{Korrespondenzadresse}

\section{Klingelhoefer}

Klinik und Poliklinik für Neurologie, Technische Universität Dresden

Fetscherstraße 74, 01307 Dresden, Deutschland Lisa.klingelhoefer@uniklinikum-dresden.de

Funding. Open Access funding provided by Projekt DEAL.

\section{Einhaltung ethischer Richtlinien}

Interessenkonflikt. L. Klingelhoefer, W. Jost, P. Odin, A. Storch, K. Ray Chaudhuri und H. Reichmann geben an, dass kein Interessenkonflikt besteht.

Für diesen Beitrag wurden von den Autoren keine Stu dien an Tieren durchgeführt. Das Studienprotokoll zur Validierungsstudie des DNMSQuest in deutscher Sprache wurde durch die zuständigen Ethikkommissionen geprüft und genehmigt (Dresden und Chemnitz: EK60022015, Rostock: A2016-0159). Alle Studienteilnehmer willigten vor Studienteilnahme schriftlich ein.

Open Access. Dieser Artikel wird unter der Creative Commons Namensnennung 4.0 International Lizenz veröffentlicht, welche die Nutzung, Vervielfältigung, Bearbeitung, Verbreitung und Wiedergabe in jeglichem Medium und Format erlaubt, sofern Sie den/die ursprünglichen Autor(en) und die Quelle ordnungsgemäß nennen, einen Link zur Creative Commons Lizenz beifügen und angeben, ob Änderungen vorgenommen wurden.

Die in diesem Artikel enthaltenen Bilder und sonstiges Drittmaterial unterliegen ebenfalls der genannten Creative Commons Lizenz, sofern sich aus der Abbildungslegende nichts anderes ergibt. Sofern das betreffende Material nicht unter der genannten Creative Commons Lizenz steht und die betreffende Handlung nicht nach gesetzlichen Vorschriften erlaubt ist, ist für die oben aufgeführten Weiterverwendungen des Ma- terials die Einwilligung des jeweiligen Rechteinhabers einzuholen.

Weitere Details zur Lizenz entnehmen Sie bitte der Lizenzinformation auf http://creativecommons.org/ licenses/by/4.0/deed.de.

\section{Literatur}

1. Group ESoDiEEC (2000) A prevalence study of primary dystonia in eight European countries. JNeurol 247(10):787-792

2. Defazio G, Jankovic J, Giel JL et al (2013) Descriptive epidemiology of cervical dystonia. Tremor Other Hyperkinet Mov. https://doi.org/10. 7916/D80C4TGJ

3. Steeves TD, Day L, Dykeman J et al (2012) The prevalence of primary dystonia: a systematic review and meta-analysis. Mov Disord 27(14):1789-1796

4. Chan J, Brin MF, Fahn S (1991) Idiopathic cervical dystonia: clinical characteristics. Mov Disord 6(2):119-126

5. Zetterberg L, Lindmark B, Soderlund A et al (2012) Self-Perceived non-motor aspects of cervical dystonia and their association with disability. JRehabil Med 44(11):950-954

6. Lewis L, Butler A, Jahanshahi M (2008) Depression in focal, segmental and generalized dystonia. JNeurol 255(11):1750-1755

7. Smit M, Kuiper A, Han V et al (2016) Psychiatric comorbidity is highly prevalent in idiopathic cervical dystonia and significantly influences healthrelated quality of life: Results of a controlled study Parkinsonism \& Related Disorders 30:7-12

8. Trotti LM, Esper CD, Feustel PJ et al (2009) Excessive daytime sleepiness in cervical dystonia. Parkinsonism \& Related Disorders 15(10):784-786

9. Consky E, Basinski A, Belle L et al (1990) Toronto western spasmodic torticollis rating scale (TWSTRS): assesment of validity and inter-rater reliability. Neurology 40 (suppl): 445

10. Muller J, Wissel J, Kemmler G et al (2004) Craniocervical dystonia questionnaire (CDQ-24): development and validation of a disease-specific quality of life instrument. J Neurol Neurosurg Psychiatry 75(5):749-753

11. Chaudhuri KR, Martinez-Martin P, Schapira AH et al (2006) International multicenter pilot study of the first comprehensive self-completed nonmotor symptoms questionnaire for Parkinson's disease: the NMSQuest study. Mov Disord 21(7):916-923

12. Martinez-Martin $P$, Rodriguez-Blazquez C, Kurtis MM et al (2011) The impact of non-motor symptoms on health-related quality of life of patients with Parkinson's disease. Mov Disord 26(3):399-406

13. Beaton DE, Bombardier C, Guillemin F et al (2000) Guidelines for the process of crosscultural adaptation of self-report measures. Spine 25(24):3186-3191

14. Guillemin F, Bombardier C, Beaton D (1993) Crosscultural adaptation of health-related quality of life measures: literature review and proposed guidelines. J Clin Epidemiol 46(12):1417-1432

15. Naidu Y, Martinez-Martin P, Rizos A et al (2011) The development of a non motor scale for craniocervical dystonia. Mov Disord 26(suppl 2):S1-S385

16. Klingelhoefer $L$, Martino D, Martinez-Martin P et al (2014) Nonmotor symptoms and focal cervical dystonia: observations from 102 patients. Basal Ganglia 4:117-120

17. Klingelhoefer $L$, Warner $T$, Martino $D$ et al (2014) Evaluation of the use of a dystonia non 
motor symptom questionnaire for craniocervical dystonia in the outpatient clinic. Mov Disord 29(Suppl 1):S1-S571 (Abs N: 1386)

18. Klingelhoefer L, Chaudhuri KR, Kamm Cet al (2019) Validation of a self-completed dystonia non-motor symptoms questionnaire. Ann Clin Transl Neurol 6(10):2054-2065

19. Comella CL, Leurgans S, Wuu J et al (2003) Rating scales for dystonia: a multicenter assessment. Mov Disord 18(3):303-312

20. Jost WH, Hefter H, Stenner A et al (2013) Rating scales for cervical dystonia: a critical evaluation of tools for outcome assessment of botulinum toxin therapy. J Neural Transm 120(3):487-496

21. Stamelou M, Edwards MJ, Hallett Met al (2012) The non-motor syndrome of primary dystonia: clinical and pathophysiological implications. Brain 135(Pt 6):1668-1681

22. Ben-Shlomo Y, Camfield L, Warner T (2002) What are the determinants of quality of life in people with cervical dystonia? J Neurol Neurosurg Psychiatry 72(5):608-614

23. Camfield L, Ben-ShlomoY, WarnerTT(2002) Impact of cervical dystonia on quality of life. Mov Disord 17(4):838-841

24. Werle RW, Takeda SY, Zonta MB et al (2014) The physical, social and emotional aspects are the most affected in the quality of life of the patients with cervical dystonia. Arq Neuropsiquiatr 72(6):405-410

25. Slawek J, Friedman A, Potulska A et al (2007) Factors affecting the health-related quality of life of patients with cervical dystonia and the impact of botulinum toxin type A injections. Funct Neurol 22(2):95-100

26. Schapira AHV, Chaudhuri KR, Jenner P (2017) Non-motor features of Parkinson disease. Nat Rev Neurosci 18(8):509
Hansen H.C., Dohmen C., Els T., Haupt W.F., Wetheimer D., Erbguth F. (Hrsg)

\section{Notfälle mit Bewusstseinsstörungen und Koma}

Interdisziplinäre Fallbeispiele und Analysen

Berlin Heidelberg: Springer-Verlag 2019, 1, 251 S., 52 schwarz-weiß, 41 Farbe Abb., (ISBN: 978-3-662-59128-4), 49,99 EUR

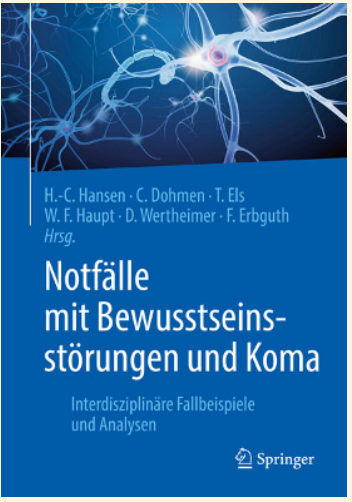

Akute Störungen des Bewusstseins stellen häufig schwierige Situationen in zentralen Notaufnahmen oder auf Intensivstationen dar. Ihr Management erfordert fundierte medizinische Kenntnisse und große Erfahrung und oft eine interdisziplinäre diagnostische und therapeutische Herangehensweise.

Die Autoren schildern anhand von 36 Kasuistiken praxisnah die differentialdiagnostischen Überlegungen bei verschiedenen neurologischen Notfallsituationen mit den Leitsymptomen Bewusstseinsstörung und Koma. Die Kasuistiken sind primär nach Altersgruppen geordnet, im Kindes- und Jugendalter beginnend, gefolgt von solchen im mittleren Lebensabschnitt bis hin zu den Älteren. Vaskuläre, metabolische, entzündliche, traumatische und andere Ursachen für Bewusstseinsstörungen werden detailliert und anschaulich abgehandelt.

Der pädagogische Ansatz liegt in einer kurzweiligen Lektüre, die fast spielerisch durch die Thematik führt, und weniger in einem Nachschlagewerk. Dramaturgisch sind die Beschreibungen unterschiedlicher Szenarien so wirklichkeitsnah geschildert, dass den Leser sofort die typische Anspannung und Erregung packt, die man sonst in der Notaufnahme oder auf der Intensivstation erlebt.

Kurz und prägnant führen die Autoren in die Thematik anhand einer Notfall-Anamnese ein. Im Verlauf erarbeiten sie anhand eines realistischen Behandlungspfades strukturiert und sachlich die abschließende Diagnose, die immer mit einer "Take Home Message" abgerundet wird. Zum Verständnis tragen insbesondere die begleitenden Labor-, elektrophysiologischen und bildgebenden Befunde bei. „Gekrönt" werden einige Kasuistiken mit Photos von pathologischen und histologischen Präparaten sowie Verweisen auf die Pharmakologie, was sehr zum Verständnis der geschilderten Szenarien beiträgt - und in dieser Form in aktuellen Publikation eher selten ist.

Beim ersten Durchlesen gewinnt man zunächst den Eindruck einer Sammlung ungewöhnlicher Fälle. Tatsächlich wird aber die Differentialdiagnose verschiedener Ursachen von Bewusstseinsstörungen umfangreich dargestellt, oft mit zunächst eindeutig erscheinenden Verdachtsdiagnosen, die dann aber doch eine überraschende Wendung nehmen.

Auch Raritäten wie die West-Nil-Virus-Enzephalomyelitis oder das CLIPPERS-Syndrom werden erwähnt. Dabei bleibt der Schreibstil ausgesprochen sachlich und zurückhaltend, wodurch der Leser zum differential-diagnostischen Nachdenken angeregt wird.

So ist dieses Buch eine lesenswerte Lektüre, etwa für die abendliche Auseinandersetzung mit der neurologischen Notfallmedizin oder z.B. zum intellektuellen Müssiggang während längerer Reisen. Es vermittelt auf höchstem Niveau elegant und gekonnt viel Detailwissen. Insgesamt ein sehr gelungenes Buch, das Freude macht! 